\title{
De-prescribing: When Less Is More in Healthcare
}

"It is an art of no little importance to administer medicines properly; but it is an art of much greater and more difficult acquisition to know when to suspend or altogether omit them" (Pinel 1809).

A S Pinel's quote illustrates, the understanding that less is Sometimes more in healthcare is not new, but there is growing energy behind efforts to advance appropriateness of care across the health sector. Ensuring the right care for each person - neither more nor less - is the goal.

In some ways, the challenge is growing over time because complex care patterns are an increasing reality in developed countries. The 2014 Commonwealth Fund survey, for instance, found that between $8 \%$ and $25 \%$ of seniors across 11 countries saw four or more doctors in the last year. And between $29 \%$ and $53 \%$ took four or more prescription medications. In both cases, Switzerland had the lowest rates and the US the highest. Likewise, the Canadian Institute for Health Information (2014) reports that nearly two-thirds of seniors took five or more prescription medications in 2012. More than one-quarter (27.2\%) had claims for 10 or more medications.

With complex care patterns comes a need for strong coordination of care. Across developed countries, patients who say that they saw four or more physicians are at least twice as likely as those with one or two physicians to report having experienced a medical mistake, medication error, or laboratory test problem in the past two years (Commonwealth Fund 2010). And in Canada in 2012, 24\% of those aged 65 and older - or more than a million people - had been prescribed a medication that was potentially inappropriate for seniors (CIHI 2014).

The statistics are clear, but there is nothing like personal experience to bring home the human costs involved. Two years ago, a relative of mine had been prescribed a complicated cocktail of medications. It was a true medication cascade, built up over time as clinicians added new drugs to address side effects generated by medications prescribed by others. Several falls, long hospitalizations, and broken bones later, she moved into long-term care. Her de-prescribing journey, initiated by a geriatrician during her last hospital stay, continues. And it has been a journey, complete with starts and stops, progress and setbacks.

A recent Canadian Institutes of Health Research meeting for stakeholders keen to improve safe and appropriate medical therapy for older men and women drew attention to the range of approaches that can be taken to advance this goal. Different strategies focus 
at individual and interpersonal, health professional, organizational, community, and public policy levels. There are guidelines on polypharmacy, the Choosing Wisely Canada campaign targeted at both the public and health professionals, decision support tools that can be integrated into practice at the point of care, policy changes that have offered additional support for health professionals to undertake medication reviews, and much more.

Advancing understanding of the effectiveness of different types of interventions to promote appropriate care given individuals' unique needs and circumstances has the potential to help shape the future. A key challenge is to increase knowledge about which of these approaches are most effective, or, perhaps more likely, what combination of interventions works best in different circumstances. This is not just true with regards to efforts to improve the appropriateness of medications, but also for other types of care.

The articles in this issue of the journal respond to challenges like this and other important issues that cut across the continuum of care. For example, Deirdre DeJean and colleagues explore the appropriateness of ambulance service use from the perspective of paramedics. Likewise, Nathalie Clavel and colleagues examine the perspectives of decision-makers on the appropriateness of total joint replacement. Other authors focus on different types of questions related to primary care, specialty services, and emergency departments. Rounding out this issue is Karen Jackson, Omenaa Boakye and Nicole Wallace's article. It touches on knowledge translation for addressing practice and policy gaps related to integrated care.

I hope that this combination offers food for thought, regardless of your particular health interest, as well as the potential for insights from outside the areas in which each of us usually work. I also invite you to join the conversation in future issues by submitting your latest research and thoughtful evidence-informed commentaries to Healthcare Policy/Politiques en santé.

JENNIFER ZELMER, PHD

Editor-in-chief

\author{
References \\ Canadian Institute for Health Information (CIHI). 2014. Drug Use among Seniors on Public Drug Programs in \\ Canada, 2012. Retrieved January 22, 2016. <https://secure.cihi.ca/free_products/Drug_Use_in_Seniors_on_ \\ Public_Drug_Programs_2012_EN_web.pdf $>$. \\ Commonwealth Fund. 2010. The Commonwealth Fund 2010 International Health Policy Survey in Eleven \\ Countries. Retrieved January 22, 2016. <http://www.commonwealthfund.org/ /media/files/publications/ \\ chartbook/2010/pdf_2010_ihp_survey_chartpack_full_12022010.pdf>. \\ Commonwealth Fund. 2014. Appendix A1: Profiles of Primary Care Organization, Payment, and Incentives \\ in Eleven Countries. Retrieved January 22, 2016. <http://www.commonwealthfund.org/ /media/files/ \\ publications/in-the-literature/2014/nov/1787_commonwealth_fund_2014_intl_survey_appendices.pdf >. \\ Pinel, P. 1809. Medico-Philosophical Treatise on Mental Alienation, Second Edition: Entirely Reworked and \\ Extensively Expanded. As translated by G. Hickish, D. Healy and L.C. Charland. (2008). Chichester, West \\ Sussex: John Wiley and Sons.
}




\section{Dé-prescrire : quand moins veut dire plus dans les services de santé}

"L'habileté du médecin consiste moins dans l'usage répété des remèdes, que dans l'art profondément combiné d'en user à propos ou de s'en abstenir » (Pinel 1809).

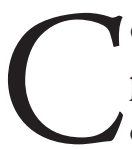

omme le montre la citation de Pinel, le principe voulant que moins peut parfois signifier plus dans les services de santé ne date pas d'hier. On observe, en effet, que de plus en plus d'énergie est consacrée aux efforts qui visent la pertinence appropriée des soins, et ce, dans tous les secteurs de la santé. L'objectif n'est ni plus ni moins que d'assurer que chaque personne reçoive les soins adéquats.

Dans un sens, ce défi prend de plus en plus d’ampleur puisque les schémas de soins complexes sont de plus en plus présents dans les pays développés. À titre d'exemple, l'enquête 2014 du Fonds du Commonwealth révèle quentre 8 et $25 \%$ des aînés de 11 pays ont consulté au moins quatre médecins au cours de l'année dernière. De plus, entre 29 et 53 \% d'entre eux ont pris au moins quatre médicaments sur ordonnance. Dans les deux cas, on observe en Suisse les taux les plus bas et aux États-Unis les taux les plus élevés. Dans le même ordre d'idée, l'Institut canadien d'information sur la santé (2014) rapporte que près des deux tiers des aînés prenaient au moins cinq médicaments sur ordonnances en 2012. Plus d'un quart (27,2 \%) des personnes âgées indiquaient prendre au moins 10 médicaments.

Les schémas de soins complexes demandent une bonne coordination des soins. Dans les pays développés, les patients qui indiquent avoir consulté au moins quatre médecins sont deux fois plus susceptibles, que ceux qui indiquent avoir consulté un ou deux médecins, de déclarer avoir fait l'objet d'une erreur médicale, d'une erreur de médication ou d'un problème d'analyse en laboratoire au cours des deux années écoulées (Fonds du Commonwealth 2010). En 2012, au Canada, 24 \% des personnes de 65 ans et plus - soit plus d'un million de personnes - ont reçu une ordonnance pour un médicament potentiellement contre-indiqué pour les personnes âgées (ICIS 2014).

Les statistiques ne mentent pas, mais il n'y a rien comme l'expérience personnelle pour en illustrer le coût humain. Il y a deux ans, une de mes parentes se trouvait devant un cocktail élaboré de médicaments sur ordonnance. Ce cocktail résultait d'une véritable montée en cascade, dans laquelle des médecins ajoutaient de nouveaux médicaments pour traiter les effets secondaires de médicaments prescrits par d'autres. Après plusieurs chutes, de longues hospitalisations et des fractures, elle a été transférée en soins de longue durée. Son travail de « dé-prescription », amorcé par un gériatre au cours de la dernière hospitalisation, est toujours en cours. Il s'agit d'un véritable périple, avec ses départs, ses arrêts, ses avancées et ses reculs.

Une réunion, organisée récemment par les Instituts de recherche en santé du Canada pour les intervenants soucieux d'améliorer la prestation de soins sécuritaires et appropriés, a fait voir 
l'étendue des démarches quon peut entreprendre pour atteindre cet objectif. Nombres de stratégies agissent aux niveaux personnel, interpersonnel, professionnel, organisationnel, communautaire ou politique. Il y a, par exemple, des directives en polypharmacie, la campagne «Choisir avec soin » qui vise autant le grand public que les professionnels de la santé, des outils d'aide à la décision quon peut intégrer directement aux points de services, des changements politiques qui offrent un soutien supplémentaire aux professionnels pour revoir la médication, et la liste est longue.

Mieux comprendre l'efficacité des divers types d'interventions visant la promotion de soins appropriés en fonction des circonstances et besoins particuliers de chacun peut nous aider à mieux planifier l'avenir. Un des principaux défi est d'approfondir les connaissances sur les démarches les plus efficaces, ou plutôt, sur la combinaison d'interventions qui fonctionne le mieux dans telle ou telle circonstance. Et cela ne concerne pas seulement l'amélioration de l'adéquation des médicaments, mais aussi celle de tout autre type de soin.

Les articles du présent numéro traitent de défis de ce genre de même que d'enjeux importants qui touchent à l'ensemble des soins. Par exemple, Deirdre DeJean et ses collègues se penchent sur la pertinence de l'utilisation de l'ambulance du point de vue des ambulanciers. Pour sa part, Nathalie Clavel et ses collègues examinent le point de vue des décideurs sur la pertinence de l'arthroplastie totale. D'autres auteurs s'intéressent à diverses questions liées aux soins primaires, aux soins des spécialistes et aux services des urgences. Le numéro se complète avec l'article de Karen Jackson, Omenaa Boakye et Nicole Wallace sur le rôle du transfert de connaissances pour combler les fossés entre les politiques et la pratique dans le contexte des soins intégrés.

J'espère que cette combinaison de sujets nourrira votre réflexion, quels que soient vos intérêts, et vous donnera des pistes provenant d'autres secteurs que le vôtre. Je vous invite également à participer aux débats de cette revue en soumettant vos derniers rapports de recherche ou des commentaires éclairés par les données probantes, pour les prochains numéros de Politiques en santé/Healthcare Policy.

JENNIFER ZELMER, PHD

Rédactrice en chef

\section{Références}

Fonds du Commonwealth. 2010. The Commonwealth Fund 2010 International Health Policy Survey in Eleven Countries. Consulté le 22 janvier 2016. <http://www.commonwealthfund.org/ /media/files/publications/ chartbook/2010/pdf_2010_ihp_survey_chartpack_full_12022010.pdf >.

Fonds du Commonwealth. 2014. Appendix A1: Profiles of Primary Care Organization, Payment, and Incentives in Eleven Countries. Consulté le 22 janvier 2016. <http://www.commonwealthfund.org/ /media/files/ publications/in-the-literature/2014/nov/1787_commonwealth_fund_2014_intl_survey_appendices.pdf >.

Institut canadien d'information sur la santé (ICIS). 2014. Utilisation des médicaments chez les personnes âgées adhérant à un régime public d’assurance-médicaments au Canada, 2012. Consulté le 22 janvier 2016. $<$ https://secure.cihi.ca/free_products/Drug_Use_in_Seniors_on_Public_Drug_Programs_2012_FR_web.pdf>. Pinel, P. 1809. Traité médico-philosophique sur l'aliénation mentale, seconde édition : entièrement refondue et très augmentée, p. XXV. Consulté le 2 février 2016. <http://gallica.bnf.fr/ark:/12148/bpt6k65654178.r=Traité\%20 médico-philosophique\%20sur\%201\%27aliénation\%20mentale>. 


\section{HEALTHCARE

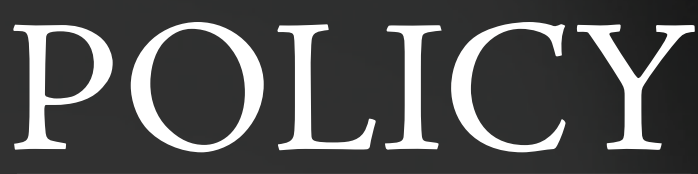 \\ Politiques de Santé}

\section{Special Issues Free for the Reader}

\section{Approaches to Accountability}

"... this set of papers raises important issues around the theme of accountability. First, it shows that accountability is still in its infancy - not because providers or organizations do not want to be accountable or that governing bodies do not want to make them accountable, but because identifying the right targets and establishing the right mechanisms to account for the utilization of healthcare resources is a complex task." - Guest Editor Jean-Louis Denis

\section{Going for the Gold}

"MCHP (among other centres) has shown that privacy concerns are not the issue; nor is it anything to do with the potential power of the analytical results. Perhaps it is this very power, though, that is at the root of the lack of progress." - Guest Editor Michael Wolfson

\section{Primary Healthcare Information System}

"This special issue of Healthcare Policy/Politiques de Santé gives readers important new information on what can be done with existing PHC data sources and the requirements for additional data sources and systems to support health system management and policy development." - Guest Editor Greg Webster

\section{Regional Training Centres}

"Canadian Health Services Research Foundation (CHSRF) and the Canadian Institutes of Health Research (CIHR) took on the basic blueprinting task when they envisioned the RTCs as a new approach to increasing capacity in applied health and nursing services research in Canada." - Guest Editor Pat Martens

\section{HealthcarePolicy.net}

